\title{
Endovascular Management of Pancreatitis-Related Hemorrhage: Single-Center Experience
}

\author{
Sunil K. Kumar ${ }^{1, \odot}$ Jagadeesh R. Singh ${ }^{1} \quad$ Mahesh T. Kumar ${ }^{1}$ \\ ${ }^{1}$ Department of Interventional Radiology, Asian Institute of \\ Gastroenterology, Davanagere, Hyderabad, Telangana, India \\ ${ }^{2}$ Department of Medical Gastroenterology, Asian Institute of \\ Gastroenterology, Hyderabad, Telangana, India \\ ${ }^{3}$ Department of Surgical Gastroenterology, Asian Institute of \\ gastroenterology, Hyderabad, Telangana, India

K.N. Nagbhushan ${ }^{1} \quad$ Nageshwar D. Reddy ${ }^{2}$

G.V. $\mathrm{RaO}^{3}$

\begin{abstract}
Address for correspondence Sunil Kumar K, MD, Department of Interventional Radiology, Asian Institute of Gastroenterology, Hyderabad 500082, Telangana, India (e-mail: drsunilkumark@yahoo.in).
\end{abstract}

\begin{abstract}
Purpose The main purpose of this article is to assess the outcomes of endovascular management of pancreatitis-related hemorrhage.

Materials and Methods Retrospective analysis of patients referred for endovascular management of pancreatitis-related bleeding from January 2010 to December 2017 was performed. Patients' demographics, clinical presentation, etiology, laboratory findings, angiography findings, details of the endovascular procedure, technical outcome, clinical outcome, and complications were assessed.

Results One hundred and five patients with a mean age of 37 years were included in this study. Splenic artery (41.7\%) was the most commonly involved vessel. Middle colic, left colic, superior mesenteric, jejunal, left inferior phrenic, and left renal subcapsular arteries were the less commonly involved vessels. Embolization was performed using coils in 72 (68.5\%) patients, $n$-butyl cyanoacrylate (n-BCA) in 21 (20\%) patients, both coil and $n$-BCA in $8(7.6 \%)$ patients, and $4(3.8 \%)$ patients underwent stent graft placement. Technical success was achieved in $98 \%(n=103)$ and clinical success in $93.2 \%(n=96)$ of patients. Rebleeding was seen in $6.8 \%(n=7)$ of patients. Six patients with rebleeding were managed by reintervention. Four patients had rebleeding from

Keywords

- pancreatitis

- hemorrhage

- pseudoaneurysm

- embolization the same vessel, and two patients had rebleeding from a different vessel. One patient died due to massive rebleeding. Minor complications were seen in $14.2 \%(n=15)$, major complications were seen in 3.8\% $(n=4)$, and mortality rate was $0.9 \%(n=1)$.

Conclusion Endovascular treatment is effective in the management of pancreatitis-related bleeding and is associated with low rebleeding rate and low mortality rate.
\end{abstract}

\section{Introduction}

Arterial hemorrhage is a rare complication of pancreatitis. The incidence of vascular complications in pancreatitis ranges from 1.2 to $15.2 \%$. The vascular complications are more common in patients with chronic pancreatitis than acute pancreatitis with a reported frequency of 7 to $10 \%$ in patients with chronic pancreatitis versus 1 to $6 \%$ in patients with acute pancreatitis., ${ }^{1,2}$ The most common vessels involved in pancreatitis-related pseudoaneurysm are the splenic artery in 30 to $50 \%$, the gastroduodenal artery (GDA) in 20 to $25 \%$, pancreaticoduodenal vessels in 20 to $25 \%$, and mesenteric, colic, and hepatic vessels in the remaining patients. ${ }^{3}$ Mortality due to arterial hemorrhage in pancreatitis can be as
DOI https://doi.org/ 10.1055/s-0040-1705290 ISSN 2457-0214.
License terms

() (1) $\ominus \circledast$ 
high as 34\% ${ }^{4-7}$ Mortality following surgical management of arterial hemorrhage varies from 20 to $50 \%$ with a rebleeding rate as high as $25 \%{ }^{7}$

Arterial hemorrhage in pancreatitis is multifactorial. In the presence of acute inflammation, the release of pancreatic enzymes leads to vessel wall weakening and erosions resulting in disruption of vessel wall and pseudoaneurysm. In pseudocysts and pancreatic collections, a combination of ischemic necrosis, infection, and the effect of the pancreatic enzymes causes vessel injury, pseudoaneurysm, and bleeding. ${ }^{5}$

Though endovascular management is the initial treatment option in most centers and is preferred over surgical management, the literature on the effectiveness of endovascular treatment is limited and the largest published data included only 37 patients. ${ }^{8,9}$ In this large population study, we report the safety and effectiveness of endovascular management in pancreatitis-related bleeding.

\section{Material and Methods}

This is a retrospective study of patients who had undergone endovascular management for pancreatitis-related bleeding at a tertiary care center. The data of all patients who received embolization or endovascular exclusion with stent grafts for pancreatitis-related bleeding between January 2010 to December 2017 were included. Ethical review was not required for this retrospective study. There were 105 patients (99 males and 6 females) with a mean age of 37 years (range: 7-73 years). Patients' demographics, clinical presentation, etiology, laboratory findings, angiography findings, details of endovascular procedure, technical outcome, clinical outcome, and complications were assessed. Computed tomography (CT) angiography was performed using a six slice multidetector CT scanner (Siemens Emotion) in all cases.

\section{Angiography and Endovascular Management}

Angiography were performed on a digital subtraction angiography system (Axiom Artis, Siemens). Angiography and endovascular interventions were performed through a right common femoral arterial approach in 104 patients and through a brachial artery approach in 1 patient. The celiac artery, superior mesenteric artery (SMA), and inferior mesenteric artery were catheterized and selective angiography performed. Super-selective catheterization of the common hepatic artery, GDA, left gastric artery, and splenic artery were performed either with a $4 \mathrm{~F}$ catheter or a $3 \mathrm{~F}$ microcatheter (Microferret, Cook Medical Inc.). The angiography images were reviewed for the presence of pseudoaneurysm, contrast extravasation, and vessel wall irregularity. The vascular abnormalities were treated either by embolization or by stent graft placement. Embolization was performed with coils of varying sizes (Cook Medical Inc.) or $n$-butyl cyanoacrylate ( $n$-BCA) (Reevox Pharma) delivered through a $4 \mathrm{~F}$ catheter for larger vessels or through a $3 \mathrm{~F}$ microcatheter for smaller vessels. Coils were the preferred embolic materials, as coil embolization is technically easy, accurate, and pseudoaneurysm can be excluded by proximal and distal coiling. Liquid embolic materials were preferred in pseudoaneurysm with difficult cannulation and in patient with multiple feeders. Stent graft was preferred in technically feasible end arteries to prevent ischemic complications. $n$-BCA was mixed with lipiodol (Guerbet) in the ratio of $1: 1,1: 2$, and $1: 3$ depending on the flow dynamics. For stent graft placement, the artery was catheterized, and access was secured with a stiff wire and a stent graft was deployed across the aneurysm or vascular abnormality. Angiography was repeated at the end of the procedure in all cases. The mean fluoroscopy time was 20 minutes (range: 15-60 minutes).

\section{Outcomes}

A technically successful embolization was defined as devascularization or cessation of blood flow to the target vascular bed. Technical success reflects immediate results and is evaluated at the completion angiography. Clinical success was defined as no rebleeding within 30 days of endovascular intervention and resolution of signs or symptoms that prompted the embolization procedure. ${ }^{10}$ Patients were monitored for immediate procedure-related complications like groin hematoma and pseudoaneurysm. Ultrasound was performed to assess for liver, splenic infarcts, and abscess. Clinical follow-up was done for any possible bowel ischemia.

\section{Results}

The demographics and clinical details of the patients are summarized in - Table 1. Acute pancreatitis was seen in 37 patients and chronic pancreatitis in 68 patients. Abdominal pain was present in 74 patients, hematemesis in 35 patients, melena in 42 patients, hematochezia in 3 patients, and hemodynamic instability in 12 patients. The mean hemoglobin level was $8.3 \mathrm{~g} / \mathrm{dL}$.

Fifty-eight patients received blood transfusion: 21 patients received blood transfusion prior to embolization and 37 patients

Table 1 Patient demographics, etiology, clinical presentation, and laboratory findings

\begin{tabular}{|l|l|}
\hline Number of patients & 105 \\
\hline Male:female & $99: 6$ \\
\hline Mean age (range) & 37 (7-73 years) \\
\hline Etiology & $37(35.2 \%)$ \\
\hline Acute pancreatitis & $68(64.7 \%)$ \\
\hline Chronic pancreatitis & \\
\hline Clinical presentation & 74 \\
\hline Pain abdomen & 35 \\
\hline Hematemesis & 42 \\
\hline Melena & 3 \\
\hline Hematochezia & 12 \\
\hline Hemodynamic instability & $8.3 \mathrm{~g} / \mathrm{dL}$ \\
\hline Mean hemoglobin & \\
\hline
\end{tabular}


following the embolization with a mean of 3.3 units of blood transfused per patient.

Conventional angiography was positive in 102 patients of which CT angiography was positive in 95 patients and negative in 7. Angiography demonstrated a pseudoaneurysm in 98 patients and contrast extravasation in 7 patients. This is compared with pseudoaneurysm in 89 patients and contrast extravasation in 6 patients on CT angiography. Both CT and conventional angiography were negative in three patients, and these three patients underwent empirical embolization of the GDA. Empirical embolization is therapeutic embolization of a vessel in which no active bleeding was identified. ${ }^{11}$ Selection of artery for empirical embolization was based on both endoscopy findings and angiography findings such as the attenuated vessel and irregular margins of the artery. Arteries involved in the pancreatitis-related hemorrhage are summarized in - Table 2.

Splenic artery was the most common involved vessel $(n=48)$ (-Fig. 1) followed by GDA $(n=29)$ (-Fig. 2). Left inferior phrenic artery $(n=2)$, left subcapsular renal artery $(n=1)$, and middle colic, left colic, and jejunal arteries (each $n=1$ ) were the uncommon arteries involved.

Details of endovascular procedure and outcomes are detailed in - Table 3. Embolization was performed in 101 patients. Coils were used in 72 patients (68.5\%) (-Figs. 1-3), $n$-BCA in 21 patients (20\%), and both coils and $n$-BCA in 8 patients $(7.6 \%)$ (-Fig. 4). Stent graft was placed in four patients (3.8\%) (-Fig. 5). Technical success was achieved in 103 (98.1\%) patients. In two patients, immediate hemostasis was not achieved, and these two patients underwent surgical management. Clinical success was achieved in 96 of 103 patients (93.2\%). Rebleeding was seen in seven patients (6.7\%). Of these seven patients with rebleeding, six patients were managed by reintervention (four patients had bleeding from the same vessel that was treated, and two patients had bleeding from a different vessel). One patient died due to massive rebleeding.

Table 2 Arteries responsible for bleeding or pseudoaneurysm (based on angiography)

\begin{tabular}{|l|l|}
\hline Artery & No. of cases (\%) \\
\hline Splenic artery & $48(45.2)$ \\
\hline GDA & $28(25.6)$ \\
\hline LGA & $9(8.2)$ \\
\hline SMA & $7(6.4)$ \\
\hline Celiac artery & $1(0.9)$ \\
\hline IPDA & $6(5.5)$ \\
\hline SPDA & $2(1.8)$ \\
\hline CHA & $2(1.8)$ \\
\hline RHA & $2(1.8)$ \\
\hline Left interior phrenic & $2(1.8)$ \\
\hline Left renal artery & $1(0.9)$ \\
\hline Middle, left colic, and jejunal artery & $1(0.9)$ \\
\hline
\end{tabular}

Abbreviations: CHA, common hepatic artery; GDA, gastroduodenal artery; IMA, inferior mesenteric artery; IPDA, inferior pancreaticoduodenal artery; LGA, left gastric artery; RHA, right hepatic artery; SMA, superior mesenteric artery; SPDA, superior pancreatoduodenal artery.
Splenic infarct was seen in 18 (17.1\%) patients, of which 15 were self-limiting. Splenic abscess and sepsis were observed in three patients. Two patients were managed conservatively, and one patient underwent splenectomy. One patient died from massive bleeding. The overall mortality rate was $1 \%$.

\section{Discussion}

Arterial hemorrhage is a rare complication of pancreatitis. The incidence of arterial complications in pancreatitis can be as high as $15.2 \%$ with a mortality rate as high as $34 \%$. The pathogenesis of arterial involvement is multifactorial and is due to destruction of vessel wall by the proteolytic enzymes released secondary to pancreatic inflammation and necrosis, and the combined effect of ischemic necrosis and proteolytic destruction of vessel wall in abscesses and pseudocysts. ${ }^{1-7}$

Noninvasive evaluation of pancreatitis-related bleeding is usually performed with CT angiography. Convention angiography is performed when CT angiography is positive, or when the bleeding persists despite a negative CT angiography. Conventional angiography has the advantage of realtime hemodynamic assessment of the vascular bed including the identification of the feeding vessel, collateral vessels, and concomitant therapeutic intervention., ${ }^{5,12,13}$ In the present study, conventional angiography was positive in 102 patients of which CT angiography was positive in 95 patients. Both CT and conventional angiography were negative in three patients and these three patients underwent empirical embolization.

The splenic artery was the most commonly involved vessel in our study. This has been previously reported in the literature. ${ }^{9,14,15}$ The less commonly involved vessels were left inferior phrenic artery (two patients), middle and left colic arteries (one patient), and left subcapsular renal artery (one patient). In literature, there are a few case reports of involvement of left renal artery, left inferior phrenic artery, and middle colic artery. ${ }^{9,15}$ Knowledge of involvement of uncommon vessels is important, as vascular abnormalities from these vessels can be missed and can lead to false negative angiography.

Arterial complications of pancreatitis are managed by embolization, stent graft placement, and surgery. Transarterial embolization has been considered as the initial treatment especially in hemodynamically stable patients. . $^{7,11,14,16}$ Surgery is reserved for patients with active bleeding, hemodynamically unstable patients, following failed embolization and for other secondary complications such as infection or extrinsic compression.7,16 Mortality rate following surgical management can range from 20 to $50 \%$, depending upon the patient's hemodynamic state, the bleeding location, and timing of the surgery. ${ }^{7}$

The reported technical success ranged from 75 to $100 \%$ and clinical success ranged from 78 to $91.9 \% .^{9,14,15,17-21}$ The technical success in our study was $98.1 \%$ and clinical success was $93.2 \%$. Coils were the preferred embolic materials. Coils were used in 72 (68.5\%) patients and $n$-BCA in 21 (20\%) patients and both in 8 (7.6\%) patients. Most studies reported use of coils for embolization of pseudoaneurysms., ${ }^{911}$ This is expected given that the coils could be precisely deployed at the intended location and the chances of nontarget 


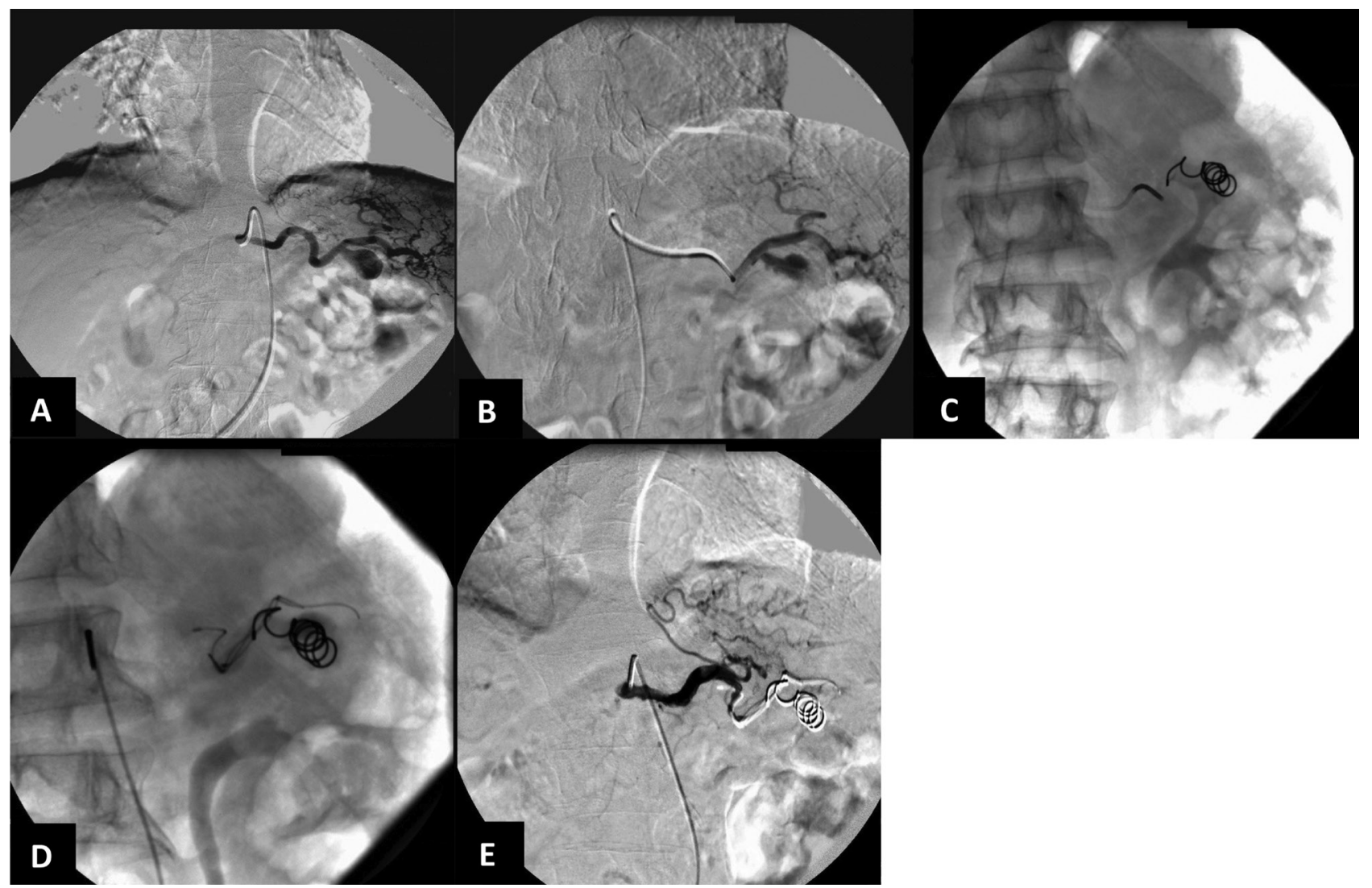

Fig. 1 A 50-year-old male with chronic pancreatitis and pain abdomen. (A and B) Splenic angiogram shows distal splenic artery pseudoaneurysm. (C and D) Coil embolization of the pseudoaneurysm and distal splenic artery. (E) Postembolization angiography showed no filling of the pseudoaneurysm.

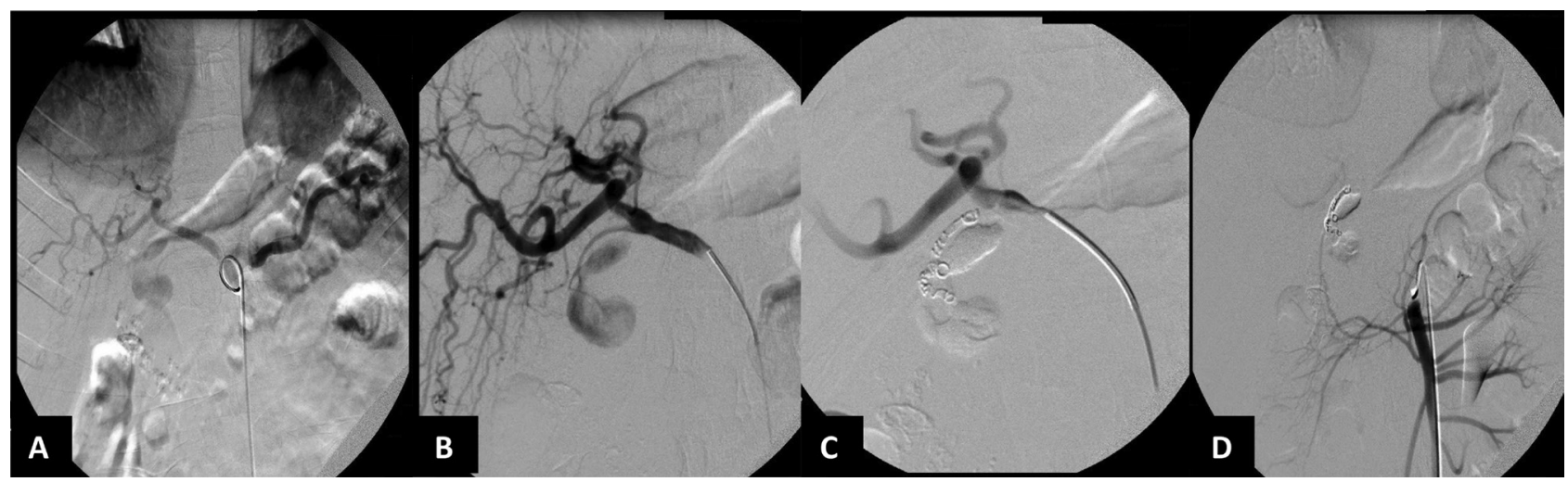

Fig. 2 A 40-year-old male patient with chronic pancreatitis and lower gastrointestinal bleeding. (A) Celiac and (B) hepatic angiography demonstrate a pseudoaneurysm of the gastroduodenal artery (GDA). Coil embolization of the GDA was performed. Postembolization (C) hepatic and (D) superior mesenteric artery angiography demonstrate no more filling of the pseudoaneurysm.

embolization would be low. A few studies have shown good outcomes with $n$-BCA..$^{22,23} n$-BCA is useful in patients with inaccessible arterial branches or aneurysms with multiple feeders or in patients with persistent aneurysm flow despite coil embolization. The main disadvantage with $n$-BCA is non target embolization. ${ }^{24}$ The reported risk of bowel ischemia following glue embolization is $5.4 \%$ in upper gastrointestinal tract and $6.1 \%$ in lower gastrointestinal tract. ${ }^{25}$ Stent graft was placed in four patients (two in splenic artery and two in
SMA). Stent graft is safe, effective, and alternate to embolization and has advantage of maintaining distal flow. ${ }^{24,26}$

Rebleeding rate following endovascular management for pancreatitis-related bleeding varies from 2.6 to $37 \%{ }^{9,11,14,15}$ Rebleeding was seen in seven (6.8\%) patients in the present study. Six patients were managed by reintervention. Four patients had rebleeding from the same vessel and two patients had bleeding from a different vessel. Etiology for rebleeding is not known. Ongoing inflammation and enzyme 
mediated destruction of the vessel might lead to continued vessel wall injury, rebleeding, or new pseudoaneurysm formation. ${ }^{9,11}$

Empirical embolization was performed in three patients. The role of empirical embolization in pancreatitis-related hemorrhage is not proven. Hyare et al performed empirical

Table 3 Details of endovascular interventions and outcomes

\begin{tabular}{|l|l|}
\hline Endovascular intervention & No. of patients (\%) \\
\hline Coils & $72(68.5)$ \\
\hline$n$-BCA & $21(20)$ \\
\hline Coils and $n$-BCA & $8(7.6)$ \\
\hline Stent graft & $4(3.8)$ \\
\hline Outcome & $103(98)$ \\
\hline Technical success & $96(93.2)$ \\
\hline Clinical success & $7(6.8)$ \\
\hline Rebleeding & $1(1)$ \\
\hline Mortality &
\end{tabular}

Abbreviation: $n$-BCA, $n$-butyl cyanoacrylate. embolization in eight patients and achieved successful hemostasis in six patients. ${ }^{11}$ Empirical embolization may be considered in angiography-negative patients with high clinical suspicion of bleeding. Selection of artery for empirical embolization is based on endoscopy findings and angiography findings such as an attenuated vessel and irregular margins of an artery.

Splenic infarct was seen in 18 (17.1\%) patients, of which 15 were self-limiting. Splenic abscess and sepsis were seen in three patients. Two patients were managed conservatively and by splenectomy in one patient. Embolization-related complications in the present study are comparable to the reported incidence ranging from 18 to 22\%.9,21,27 Mortality rate following endovascular management varies from 0 to $20 \%{ }^{10-12,14,15,17}$ Mortality rate in the present study was $1 \%$.

Our study has significant limitations. This is a retrospective study and included only patients who underwent embolization procedure at one institution. We did not include patients who underwent surgical management as the first option for pancreatitis-related bleeding. In addition, the outcomes were limited to 30 days.
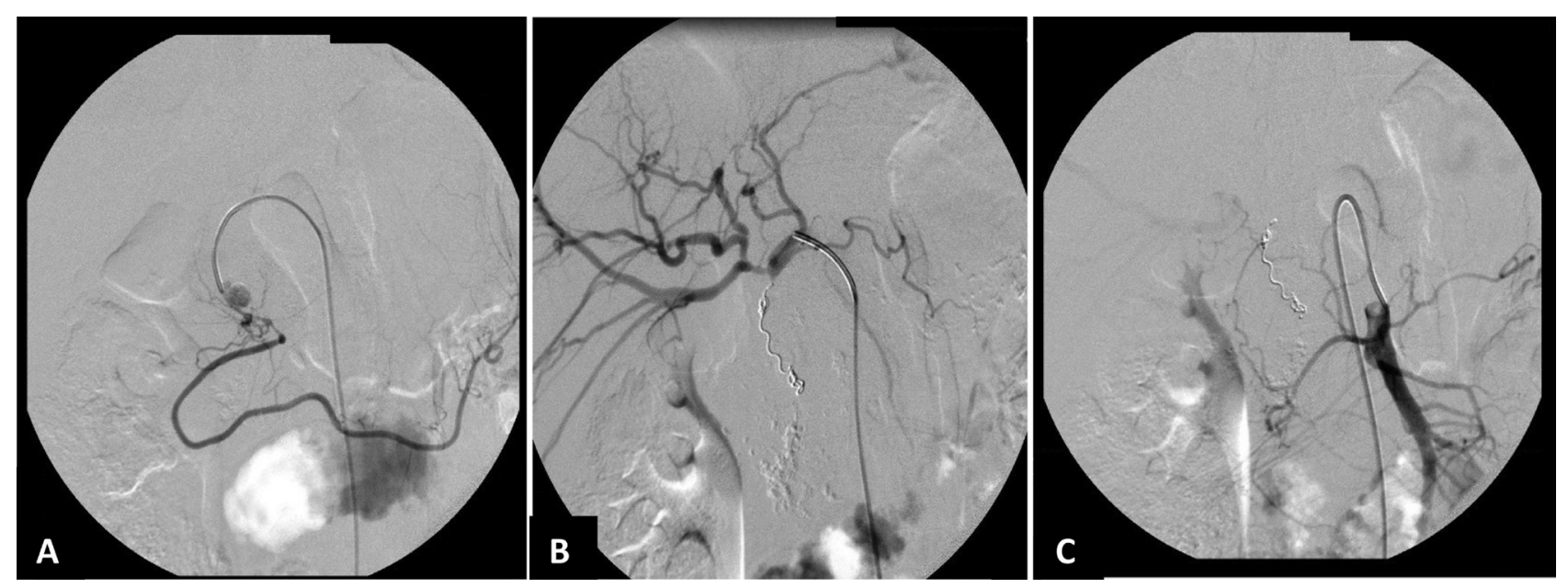

Fig. 3 A 44-year-old male patient with chronic pancreatitis and upper gastrointestinal bleeding. (A) Selective angiography of gastroduodenal artery showing pseudoaneurysm from the pancreaticoduodenal arcade. Coil embolization of superior pancreaticoduodenal artery was performed. Postembolization (B) hepatic and (C) superior mesenteric artery angiography show no pseudoaneurysm.

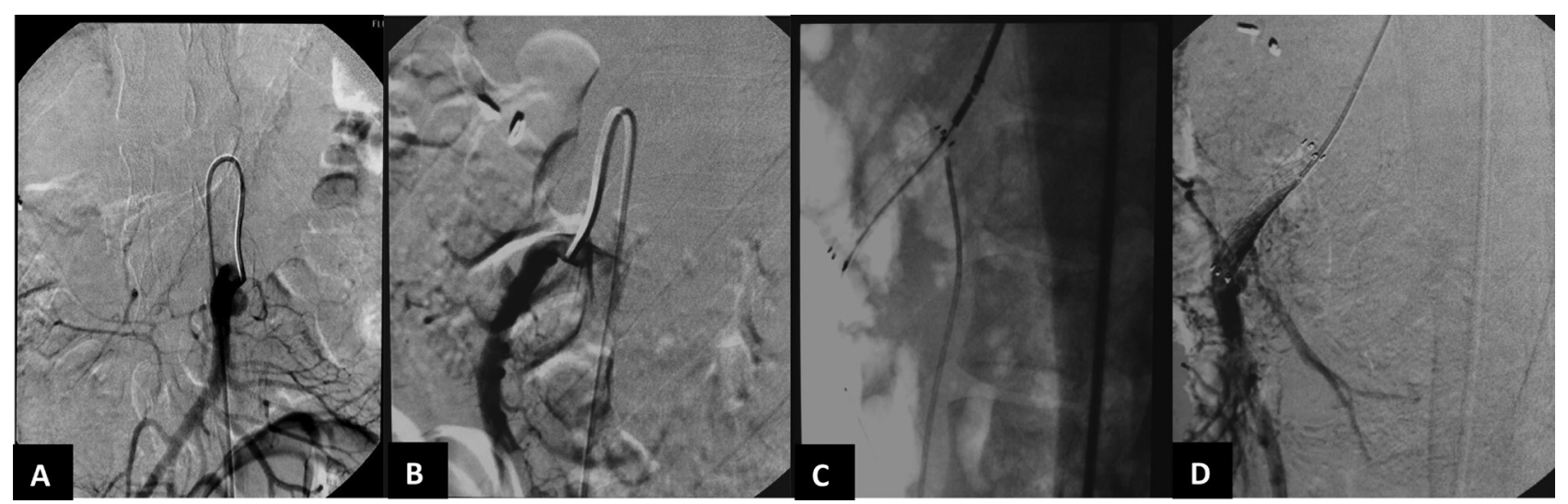

Fig. 5 A 32-year-old male patient with chronic pancreatitis and pain abdomen. (A and B) Superior mesenteric artery (SMA) angiography showing pseudoaneurysm arising from the SMA. (C) A stent graft was placed to exclude the pseudoaneurysm. (D) Post-treatment angiography showed no pseudoaneurysm. 


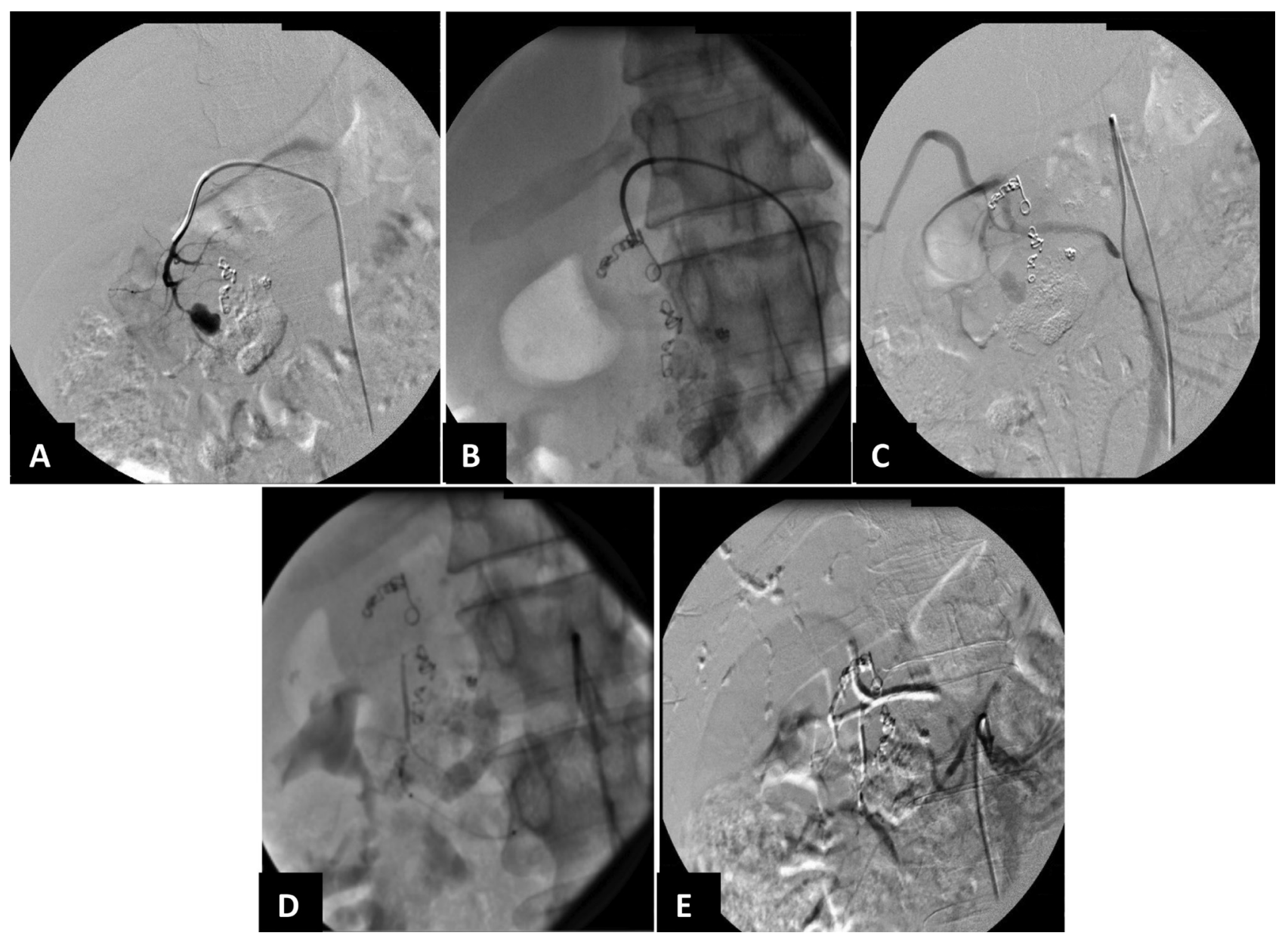

Fig. 4 A 35-year-old male patient with chronic pancreatitis and a pseudoaneurysm of the pancreaticoduodenal arcade on computed tomography. (A) Selective gastroduodenal artery angiogram showed pseudoaneurysm of the inferior pancreaticoduodenal artery (IPDA). (B) Coil embolization of the IPDA was performed. (C) Superior mesenteric artery (SMA) angiography showed refilling of the pseudoaneurysm. (D) Embolization was performed with n-butyl cyanoacrylate. (E) Postembolization SMA angiography showed no refilling of the pseudoaneurysm.

\section{Conclusion}

Endovascular management is safe and effective in the management of pancreatitis-related hemorrhage. A high technical and clinical success, low rebleeding rates, low complication, and low mortality rates in this study provide further support for endovascular therapy in pancreatitisrelated hemorrhage.

\section{Conflict of Interest}

None.

\section{References}

1 Barge JU, Lopera JE. Vascular complications of pancreatitis: role of interventional therapy. Korean J Radiol 2012;13(Suppl 1): S45-S55

2 Mallick IH, Winslet MC. Vascular complications of pancreatitis. JOP 2004;5(5):328-337

3 Evans RPT, Mourad MM, Pall G, Fisher SG, Bramhall SR. Pancreatitis: preventing catastrophic haemorrhage. World J Gastroenterol 2017;23(30):5460-5468

4 Sharma PK, Madan K, Garg PK. Hemorrhage in acute pancreatitis: should gastrointestinal bleeding be considered an organ failure? Pancreas 2008;36(2):141-145
5 Flati G, Andrén-Sandberg A, La Pinta M, Porowska B, Carboni M. Potentially fatal bleeding in acute pancreatitis: pathophysiology, prevention, and treatment. Pancreas 2003;26(1):8-14

6 Bergert H, Hinterseher I, Kersting S, Leonhardt J, Bloomenthal A, Saeger HD. Management and outcome of hemorrhage due to arterial pseudoaneurysms in pancreatitis. Surgery 2005;137(3):323-328

7 Bergert H, Dobrowolski F, Caffier S, Bloomenthal A, Hinterseher I, Saeger HD. Prevalence and treatment of bleeding complications in chronic pancreatitis. Langenbecks Arch Surg 2004;389(6):504-510

8 Kirby JM, Vora P, Midia M, Rawlinson J. Vascular complications of pancreatitis: imaging and intervention. Cardiovasc Intervent Radiol 2008;31(5):957-970

9 Kim J, Shin JH, Yoon H-K, et al. Endovascular intervention for management of pancreatitis-related bleeding: a retrospective analysis of thirty-seven patients at a single institution. Diagn Interv Radiol 2015;21(2):140-147

10 Drooz AT, Lewis CA, Allen TE, et al; Society of Interventional Radiology Standards of Practice Committee. Quality improvement guidelines for percutaneous transcatheter embolization. J Vasc Interv Radiol 2003;14(9 Pt 2, suppl):S237-S242

11 Hyare H, Desigan S, Brookes JA, Guiney MJ, Lees WR. Endovascular management of major arterial hemorrhage as a complication of inflammatory pancreatic disease. J Vasc Interv Radiol 2007;18(5):591-596 
12 Jesinger RA, Thoreson AA, Lamba R. Abdominal and pelvic aneurysms and pseudoaneurysms: imaging review with clinical, radiologic, and treatment correlation. Radiographics 2013;33(3):E71-E96

13 Saad NE, Saad WE, Davies MG, Waldman DL, Fultz PJ, Rubens DJ. Pseudoaneurysms and the role of minimally invasive techniques in their management. Radiographics 2005;25(Suppl 1): S173-S189

14 Kulkarni CB, Moorthy S, Pullara SK, Prabhu NK, Kannan RR, Nazar PK. Endovascular management of pancreatitis related pseudoaneurysms - a single center experience. Gastrointestinal Intervention 3(1):46-53

15 Boudghène F, L'Herminé C, Bigot JM. Arterial complications of pancreatitis: diagnostic and therapeutic aspects in 104 cases. J Vasc Interv Radiol 1993;4(4):551-558

16 Carr JA, Cho JS, Shepard AD, Nypaver TJ, Reddy DJ. Visceral pseudoaneurysms due to pancreatic pseudocysts: rare but lethal complications of pancreatitis. J Vasc Surg 2000;32(4):722-730

17 Vander Mijnsbrugge W, Laleman W, Van Steenbergen W, Heye S, Verslype C, Maleux G. Long-term clinical and radiological outcome of endovascular embolization of pancreatitis-related pseudoaneurysms. Acta Radiol 2017;58(3):316-322

18 Nicholson AA, Patel J, McPherson S, Shaw DR, Kessel D. Endovascular treatment of visceral aneurysms associated with pancreatitis and a suggested classification with therapeutic implications. J Vasc Interv Radiol 2006;17(8):1279-1285

19 Deshmukh H, Rathod K, Garg A, Sheth R, Kulkarni S. Transcatheter embolization as primary treatment for visceral pseudoaneurysms in pancreatitis: clinical outcome and imaging follow up. Indian J Gastroenterol 2004;23(2):56-58
20 Balachandra S, Siriwardena AK. Systematic appraisal of the management of the major vascular complications of pancreatitis. Am J Surg 2005;190(3):489-495

21 Kalva SP, Yeddula K, Wicky S. Fernandez del Castillo C, Warshaw AL. Angiographic intervention in patients with a suspected visceral artery pseudoaneurysm complicating pancreatitis and pancreatic surgery. Arch Surg 2011;146(6):647-652

22 Yamakado K, Nakatsuka A, Tanaka N, Takano K, Matsumura $\mathrm{K}$, Takeda K. Transcatheter arterial embolization of ruptured pseudoaneurysms with coils and n-butyl cyanoacrylate. J Vasc Interv Radiol 2000;11(1):66-72

23 Izaki K, Yamaguchi M, Kawasaki R, Okada T, Sugimura K, Sugimoto K. N-butyl cyanoacrylate embolization for pseudoaneurysms complicating pancreatitis or pancreatectomy. J Vasc Interv Radiol 2011;22(3):302-308

24 Chadha M, Ahuja C. Visceral artery aneurysms: diagnosis and percutaneous management. Semin Intervent Radiol 2009;26(3):196-206

25 Kim PH, Tsauo J, Shin JH, Yun SC. Transcatheter arterial embolization of gastrointestinal bleeding with N-butyl cyanoacrylate: a systematic review and meta-analysis of safety and efficacy. J Vasc Interv Radiol 2017;28(4):522-531.e5

26 Boufi M, Belmir H, Hartung O, Ramis O, Beyer L, Alimi YS. Emergency stent graft implantation for ruptured visceral artery pseudoaneurysm. J Vasc Surg 2011;53(6):1625-1631

27 Mauro MA, Jaques P. Transcatheter management of pseudoaneurysms complicating pancreatitis. J Vasc Interv Radiol $1991 ; 2(4): 527-532$ 